\title{
The acute effects of a single session of expiratory muscle strength training on blood pressure, heart rate, and oxygen saturation in healthy adults
}

\author{
Helena Laciuga $^{1 *}$, Paul Davenport ${ }^{2}$ and Christine Sapienza ${ }^{1,3}$ \\ ${ }^{1}$ Department of Speech, Language, and Hearing Sciences, University of Florida, Gainesville, FL, USA \\ ${ }^{2}$ Department of Physiological Sciences, College of Veterinary Medicine, University of Florida, Gainesville, FL, USA \\ ${ }^{3}$ Malcom Randall VA, Brain Rehabilitation Research Center, Gainesville, FL, USA
}

\section{Edited by:}

Bill J. Yates, University of Pittsburgh, USA

\section{Reviewed by:}

Samuel Verges, Institut National pour la Science et la Recherche en

Médecine - Université Joseph

Fourier, France

Hans Degens, Manchester

Metropolitan University, UK

\section{*Correspondence:}

Helena Laciuga, Department of

Speech, Language, and Hearing Sciences, University of Florida,

Laryngeal Function Lab, 21 Dauer Hall, Gainesville, FL 32611, USA.

e-mail:hlaciuga@ufl.edu
Expiratory muscle strength training (EMST) is a rehabilitative program that has been tested for outcomes related to respiratory muscle strength, cough, swallow, and voice function in healthy young adult, elderly individuals, and in patients with progressive neurodegenerative disease. Because EMST has been used in patient care, the associated cardiovascular responses during EMST are of importance. This study investigated the changes in systolic blood pressure (SBP), diastolic blood pressure (DBP), heart rate (HR), and oxygen saturation $\left(\mathrm{SpO}_{2}\right)$ during one session of EMST in healthy, young adults as a preliminary study of device safety. Thirty-one participants completed a single session of 25 trials with the EMST device. Valsalva maneuvers were performed at the beginning and at the end of the EMST trials for task comparison. The SBP, DBP, HR, and $\mathrm{SpO}_{2}$ were recorded the baseline and after completing the following tasks: a Valsalva maneuver, 12 trials using the EMST device, 13 trials using the EMST device, and 5 min of rest following the EMST session. A mixed linear model tested for changes across the six time points. The results indicated no significant change of $\mathrm{SBP}, \mathrm{DBP}, \mathrm{HR}$, or $\mathrm{SpO}_{2}$ during or following the EMST trials or after performing the Valsalva maneuver. The results suggest that EMST does not elicit significant fluctuations of blood pressure, $\mathrm{HR}$, and $\mathrm{SpO}_{2}$ in healthy young adults even when considering the effects of covariates on the outcomes measures.

Keywords: expiratory muscle strength training, cardiovascular responses, Valsalva maneuver, maximum expiratory pressure, exercise

\section{INTRODUCTION}

Expiratory muscle strength training (EMST) improves expiratory muscle force generating capacity to increase maximum expiratory driving pressure (MEP). EMST elicited increases in MEP has been reported to increase mechanical parameters during cough (Wingate et al., 2007; Pitts et al., 2009) and to support traditional voice therapy in the remediation of vocal problems (Wingate et al., 2007). Studies provide evidence of the benefits of EMST as a treatment for increasing MEP generation in those with Parkinson's disease, multiple sclerosis, (Chiara et al., 2006), as well as both sedentary and healthy elderly individuals (Baker et al., 2005; Kim et al., 2009), wind instrumentalists (Sapienza et al., 2002), professional voice users (Wingate et al., 2007), and healthy young adults (Baker et al., 2005). EMST is a potential treatment for reducing penetration and aspiration in persons with Parkinson's Disease as it increases submental muscle activity which plays a major role in hyolaryngeal complex movement during swallow (Troche et al., 2009).

It has been proposed that EMST could assist in improving respiratory function and reducing pulmonary complications in persons post stroke (Kim and Sapienza, 2005; Harraf et al., 2008). The study of Harraf et al. (2008) suggests that cortico-respiratory pathways from the affected hemisphere are disrupted in acute ischemic stroke and are associated with impairments of expiratory muscle function causing a weak cough, which impacts airway clearance and increases the risk of pneumonias with aspiration. Since a majority of stroke patients are at risk of developing cardiovascular disease and suffer from hypertension (Harraf et al., 2008), it is important to assess the cardiovascular responses to EMST prior to the application of the program with this patient group.

Expiratory muscle strength training uses a pressure threshold device (Sapienza et al., 2002; Kim et al., 2009) with a calibrated pressure relief valve housed inside the device. A sufficient expiratory pressure generation is necessary to open the pressure relief valve. The initial occlusion of the airway until the valve opens creates an isometric load to the muscles responsible for forced expiration. If the expiratory pressure produced by the user is insufficient to open the valve immediately, the user increases their physical exertion when directing the expiratory flow against the closed valve. During this effort, the intrathoracic pressure increases, and the effect may be similar to a sub-maximal Valsalva maneuver.

The Valsalva maneuver involves vocal fold closure at the end of a deep inspiration followed by physical exertion such as required during a bowel movement, or lifting a heavy weight (Metzger and Therrien, 1990). Studies have shown that prolonged breath holding and bearing down accompanied by vocal fold closure during 
activities such as weight lifting (Narloch and Brandstater, 1995), some wind instrument playing (Elghozi et al., 2008), or the supraglottic and super-supraglottic swallowing maneuvers (Levin, 1966; Chaudhuri et al., 2002) may cause a temporary but significant elevation of blood pressure (BP) as well as changes in heart rate (HR), similar to the Valsalva maneuver.

A Valsalva maneuver completed during a cardiac function test typically requires a 20-s maximum expiratory effort (Luster et al., 1996; Tiecks et al., 1996). Because a Valsalva maneuver is associated with BP and HR fluctuations related to blood flow changes and blood vessels constriction and re-expansion, which could cause the mobilization of venous thrombi, bleeding, ventricular arrhythmias, and asystole, it may be contraindicated for the patients with severe coronary artery disease, acute myocardial infarction, or moderate to severe hypovolemia (Baas et al., 2002). The EMST maneuver (Sapienza et al., 2002; Baker et al., 2005) does not require complete vocal fold closure, but involves the buildup of intraoral and intrathoracic pressures behind the closed pressure relief valve comparable to the intrathoracic pressure created during a sub-maximal Valsalva maneuver.

The contractions of thoracic and abdominal muscles used during EMST are brief and repetitive, involve rhythmic isometric and isotonic exercise components, and may be associated with an increase of intrathoracic pressure potentially similar to a Valsalva maneuver (Chaudhuri et al., 2002; McConnell and Romer, 2004). As such, the cardiovascular responses to EMST should be assessed. This study tested the effects of a single session of EMST on BP, $\mathrm{HR}$, and oxygen saturation $\left(\mathrm{SpO}_{2}\right)$ levels in young healthy adults. It was hypothesized that the fluctuations of $\mathrm{BP}, \mathrm{HR}$, and $\mathrm{SpO}_{2}$ during EMST trials would not exceed a normal range because of the short duration of approximately $2 \mathrm{~s}$ of the isometric phase of the EMST expiratory effort. We reasoned that EMST may exert similar effects on individuals with cardiovascular disease which should be considered when assessing the safety of EMST for this population.

\section{MATERIALS AND METHODS}

The study was conducted at the University of Florida, Gainesville, FL, Department of Speech, Language, and Hearing Sciences. University of Florida Institutional Review Board (IRB) approval (5002010) was obtained prior to study initiation and each participant signed an informed consent. A group of 31 healthy, young, nonsmoking adults ( 24 women and 7 men), between the ages of 18 and 30 years participated in the study.

The following exclusionary criteria were established: pregnancy as verified by a pregnancy test prior to enrollment, a body mass index (BMI) less than $18 \mathrm{~kg} / \mathrm{m}^{2}$ and greater than $33 \mathrm{~kg} / \mathrm{m}^{2}$, resting systolic blood pressure (SBP) less than $90 \mathrm{mmHg}$ and greater than $140 \mathrm{mmHg}$, resting diastolic blood pressure (DBP) less than $65 \mathrm{mmHg}$, and greater than $90 \mathrm{mmHg}$ (Joint National Committee on Prevention, Detection, Evaluation, and Treatment of High Blood Pressure, 1997), a fluctuating BP defined as three consecutive readings varying by $10 \%$ or more, and a resting $\mathrm{SpO}_{2}$ less than 95\% (Manley et al., 2001). Other exclusionary criteria included diagnosis and/or treatment for cardiovascular disease, respiratory disease that included but were not limited to: asthma, chronic obstructive pulmonary disease, and head and neck cancer within
1 year prior to the study. Participants were asked to avoid any intense physical activities prior to enrollment and advised not to consume caffeine containing products at least $2 \mathrm{~h}$ prior the study.

The measurements of BP and HR were obtained using a standardized electronic sphygmomanometer (CVS/pharmacy Automatic Blood Pressure Monitor) with an automatically pumped cuff wrapped around the left arm of the participant. $\mathrm{SpO}_{2}$ was measured using a model 8000AA-3 Adult Articulated Finger Clip Sensor (NONIN Medical, Inc.) connected to NONIN Pulse Oximeter (NONIN Medical, Inc.) and attached to the middle finger of the participant's right hand. Pulse was obtained using a MP100 Pulse Transducer attached to the index finger of the same hand. Pulse and $\mathrm{SpO}_{2}$ were measured continuously and recorded by PowerLab Version 7 and LabChart 7 software (ADInstruments). A digital manometer (MP01 mouth pressure meter; Micro Direct Inc.) was use to measure MEP (Baker et al., 2005) which is an indirect measure of expiratory muscle strength. The exercise device (EMST 150, Aspire Products) was used during the experimental session. All the measurements were taken while the participant remained in a seated position. The laboratory was maintained at temperatures between 22 and $24^{\circ} \mathrm{C}$, and the measurements were taken in the afternoon, at approximately the same time of a day for each participant.

Two samples of the participant's $\mathrm{BP}, \mathrm{HR}$, and $\mathrm{SpO}_{2}$ were measured at baseline, and the average of the two measures was considered the baseline value. The participant performed a Valsalva maneuver for $5 \mathrm{~s}$ according to instructions given by the investigator prior to the task. The instructions included the following steps: (1) take a deep breath, (2) hold your breath, (3) bear down as during a bowel movement. The 5-s duration of the Valsalva maneuver was controlled by the investigator with the use of a stopwatch. BP, HR, $\mathrm{SpO}_{2}$ were measured immediately after completing the Valsalva maneuver. During the next part of the study, MEP was measured. After a deep inhalation, the participant performed a forceful exhalation into a disposable mouthpiece attached to the manometer. This test was repeated at least three times, and each time MEP was recorded. The mean was calculated for the three highest values varying by $5 \%$ of one another. Following calculation of average MEP, the threshold pressure of the EMST device was set at 75\% of the mean MEP values as described above.

After the baseline data were collected the participant completed tasks using the EMST device. The participant was instructed to perform a deep inhalation followed by a forceful exhalation into the mouthpiece of the EMST device to open the pressure relief valve inside the device. The participant was asked to stop generating the expiratory pressure once the valve opened, which was noticeable by a hissing sound produced by the air passing through the device. The initiation and the termination of the expiratory effort were controlled by the investigator who gave the commands to the participant: "on" to begin and "off" to stop generating the expiratory pressure. The participant first completed 12 breaths into the device, and $\mathrm{BP}, \mathrm{HR}$, and $\mathrm{SpO}_{2}$ were measured immediately after the 12 trials were finished. Following these measurements, 13 remaining breaths into the EMST device were completed, and another set of measurements of $\mathrm{BP}, \mathrm{HR}$, and $\mathrm{SpO}_{2}$ were taken immediately after the task. $\mathrm{HR}$ and $\mathrm{SpO}_{2}$ were also measured continuously as the participant was completing all EMST trials. The 
participants were allowed a 5-min rest following completion of all tasks, and after the 5-min rest, the measures of $\mathrm{BP}, \mathrm{HR}$, and $\mathrm{SpO}_{2}$ were recorded. A Valsalva maneuver was performed for $5 \mathrm{~s}$, and $\mathrm{BP}, \mathrm{HR}$, and $\mathrm{SpO}_{2}$ measurements were again recorded. A single study session never exceeded $45 \mathrm{~min}$, and the last measurements of $\mathrm{BP}, \mathrm{HR}$, and $\mathrm{SpO}_{2}$ were recorded approximately $8 \mathrm{~min}$ after the EMST session. Since the cardiovascular responses to the last task (a Valsalva maneuver) fell within the normal limits, further measurements were not obtained, and the cardiovascular responses did not require any additional monitoring.

The data collected during the continuous measurements of each participant's $\mathrm{SpO}_{2}$ and $\mathrm{HR}$ were displayed as a waveform and saved using the LabChart 7 software for the further analysis. The time of each task was recorded by inserting a marker line on the waveform at the beginning and at the end of each task. The numeric values of $\mathrm{BP}, \mathrm{HR}$, and $\mathrm{SpO}_{2}$ were stored in a spreadsheet (Microsoft Office Excel 2007). A software statistical package (SAS version 9.1) was used to generate descriptive statistics of frequencies, means, and SDs for the variables in the data set. For each of the four outcome measures, SAS Proc MIXED was used to create a mixed linear model to test for changes across the six time points. In all models, the independent variable was task, and included were the covariates of: sex, BMI, and MEP. The analysis included all interactions among covariates for each outcome across whole data set. Additional analyses were performed separately for male and female participants. To determine the statistical power of the study, the SD of the differences between a particular task at baseline and after the Valsalva 1 task were used, and the smallest effect size that the study could have detected was determined with at least $90 \%$ power using paired-samples $t$-tests.

\section{RESULTS}

Table 1 shows the mean demographic data for the participants.

The following terms were used describe the experimental tasks performed by the participants: Baseline: the mean of two consecutive measurements taken prior to completing the tasks in the study; Valsalva 1: the Valsalva maneuver performed prior to the use of EMST device; Phase 1: the first phase of EMST including 12 trials using the EMST device; Phase 2: the second phase of EMST including 13 trials using the EMST device; Rest: the 5-min rest period after completing all 25 EMST trials; Valsalva 2: the Valsalva maneuver performed 5 min after completing EMST.

Mean durations of the expiratory effort and rest periods during the EMST trials were calculated for each participant as well as for the entire group. The mean expiratory exertion duration for all participants was $1.25 \mathrm{~s}$, the mean rest period between the expiratory exertions was $7.7 \mathrm{~s}$, and the mean duration of EMST session was $3 \mathrm{~min}$. $26.2 \mathrm{~s}$. There was no significant variation across tasks on any outcome measurements.

\section{SYSTOLIC BLOOD PRESSURE}

Systolic blood pressure was normally distributed across all data points with a mean of $107.7 \mathrm{mmHg}$ and a SD of $9.9 \mathrm{mmHg}$ (Figure 1). The minimum value of SBP was $89 \mathrm{mmHg}$ and the maximum was $138 \mathrm{mmHg}$. SBP did not change significantly across tasks $(p=0.1464)$. Sex had a significant effect on SBP $(F=34.06 ; \mathrm{df}=1,176 ; p<0.0001)$, with women tending to have lower mean SBP across all tasks. Mean SBP for men in the study was $118 \mathrm{mmHg}(\mathrm{SD}=10.66)$. Mean SBP for women was $105 \mathrm{mmHg}$ $(\mathrm{SD}=7.40)$. BMI had a significant effect on $\mathrm{SBP}(F=6.37$; $\mathrm{df}=1,176 ; p=0.0125$ ), with SBP increasing with BMI. Mean SBP is estimated to increase by 0.61 points for each additional point of BMI $(95 \% \mathrm{CI}=[0.136,1.083])$.

\section{DIASTOLIC BLOOD PRESSURE}

Diastolic blood pressure was normally distributed across all data points with mean of $75 \mathrm{mmHg}$ and SD of $6.8 \mathrm{mmHg}$ (Figure 2). The minimum was $61 \mathrm{mmHg}$ and the maximum was $98 \mathrm{mmHg}$. DBP did not change significantly across tasks $(p=0.8261)$. Sex had a significant effect on DBP $(F=9.42 ; \mathrm{df}=1,176 ; p=0.0025)$, with women tending to have lower mean DBP across all tasks. Mean DBP for men in the study was $79.1 \mathrm{mmHg}(\mathrm{SD}=6.92)$. Mean DBP for women was $74 \mathrm{mmHg}(\mathrm{SD}=6.19)$. BMI had a significant effect on DBP $(F=6.22 ; \mathrm{df}=1,176 ; p=0.0135)$, with DBP increasing with BMI. Mean DBP is estimated to increase by $0.48 \mathrm{mmHg}$ for each additional point of $\mathrm{BMI}(95 \% \mathrm{CI}=[0.103$, $0.859])$.

\section{HEART RATE}

Heart rate was normally distributed across all data points (Figure 3).

The overall mean was $76 \mathrm{bpm}$ with a SD of $10.6 \mathrm{bpm}$. HR did not differ significantly across the six tasks $(p=0.4501)$. BMI had a highly significant effect on $\operatorname{HR}(F=16.06 ; \mathrm{df}=1,176 ; p<0.0001)$, with HR increasing with BMI. On average, mean HR increased by $1.2 \mathrm{bpm}$ for each additional point of BMI $(95 \% \mathrm{CI}=[0.641$, $1.867])$. MEP had a significant effect on $\operatorname{HR}(F=7.09$; $\mathrm{df}=1,176$; $p=0.0085)$ with HR decreasing by $0.098 \mathrm{bpm}$ for each additional $\mathrm{cmH}_{2} \mathrm{O}$ of $\operatorname{MEP}(95 \% \mathrm{CI}=[-0.170,-0.026])$.

Table 1 | Mean demographic data.

\begin{tabular}{|c|c|c|c|c|c|c|c|c|}
\hline \multirow[t]{2}{*}{ Demographics } & \multicolumn{4}{|c|}{ Women $(N=24)$} & \multicolumn{4}{|c|}{$\operatorname{Men}(N=7)$} \\
\hline & Mean & SD & Min & Max & Mean & SD & Min & Max \\
\hline BMI $\left(k g / m^{2}\right)$ & 21.5 & 2.02 & 18.0 & 25.7 & 23.6 & 3.9 & 19.4 & 28.9 \\
\hline $\operatorname{MEP}\left(\mathrm{cmH}_{2} \mathrm{O}\right)$ & 79.0 & 17.59 & 60.0 & 119.0 & 114.4 & 30.3 & 79.0 & 164.0 \\
\hline
\end{tabular}

$N$, number of participants; SD, standard deviation; Min, minimum; Max, maximum; BMI, body mass index; MEP, maximum expiratory pressure; $y$, year. 

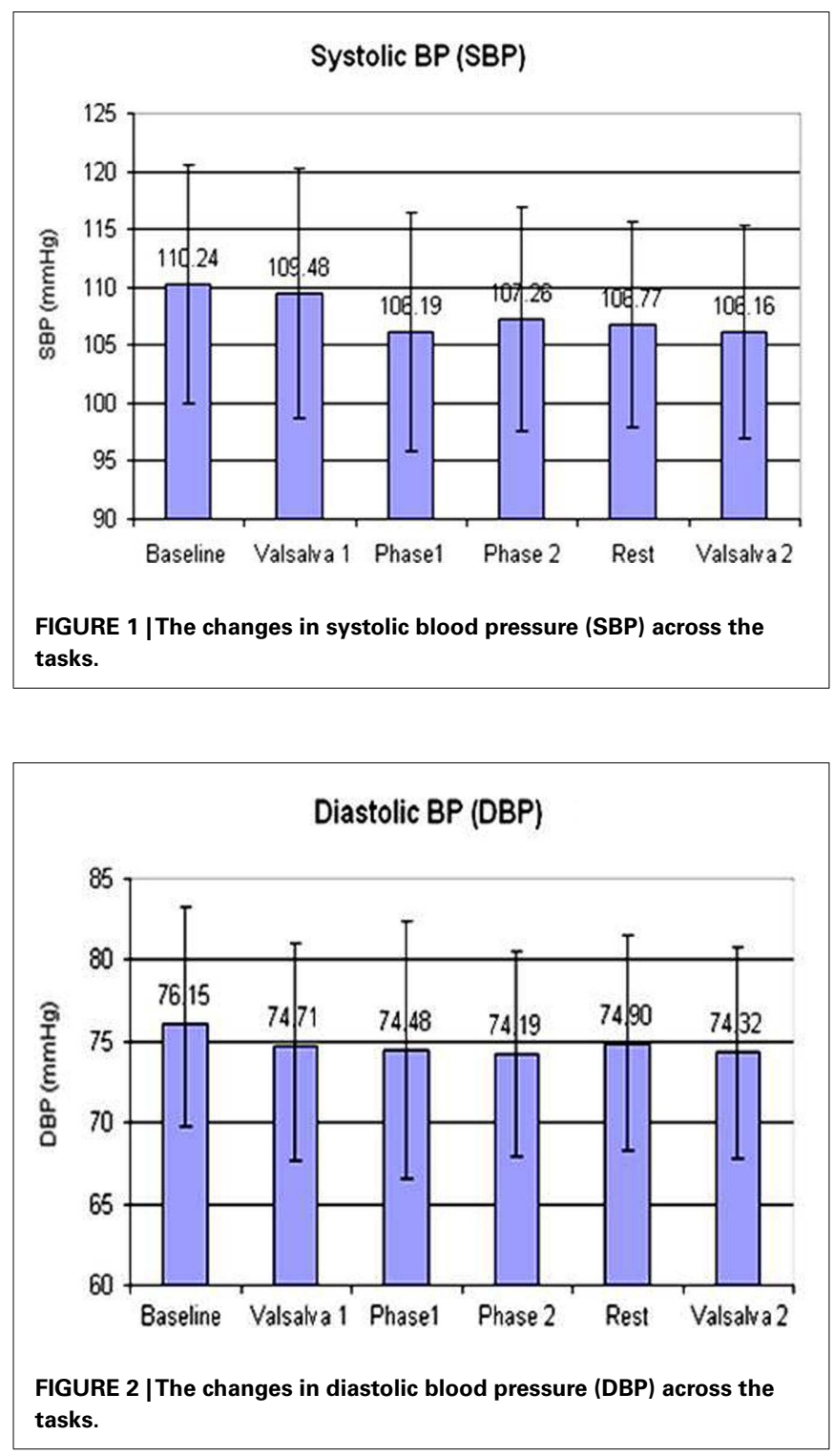

\section{PERCENT OXYGEN SATURATION}

Oxygen saturation was mildly skewed to the left with mean of 98.5\% and SD of 1.29 (Figure 4). The minimum was 93\% (after 5 min rest following the completion of EMST trials) and the maximum value was $100 \%$. Tasks performed during the study did not have a significant effect on $\mathrm{SpO}_{2}(p=0.0872)$. Sex had a significant effect on $\mathrm{SpO}_{2}(F=4.60 ; \mathrm{df}=1,176 ; p=0.0334)$, with women tending to have higher mean $\mathrm{SpO}_{2}$ across all tasks. Mean $\mathrm{SpO}_{2}$ for men in the study was $98 \%(\mathrm{SD}=1.36)$, for women was $99 \%(\mathrm{SD}=1.25)$. BMI had a significant effect on $\mathrm{SpO}_{2}(F=8.75$; $\mathrm{df}=1,176 ; p=0.0035)$, with $\mathrm{SpO}_{2}$ decreasing with increasing BMI. Mean $\mathrm{SpO}_{2}$ was estimated to decrease by $0.11 \%$ for each additional point of BMI increase $(95 \% \mathrm{CI}=[-0.185,-0.0375])$. MEP had a significant effect on $\mathrm{SpO}_{2}(F=4.40 ; \mathrm{df}=1,176$; $p=0.0375$ ), with $\mathrm{SpO}_{2}$ increasing with the increase of MEP. On average, mean $\mathrm{SpO}_{2}$ is estimated to increase by $0.0094 \%$ for each addition $\mathrm{cmH}_{2} \mathrm{O}$ of $\mathrm{MEP}(95 \% \mathrm{CI}=[0.000613,0.0182])$. The

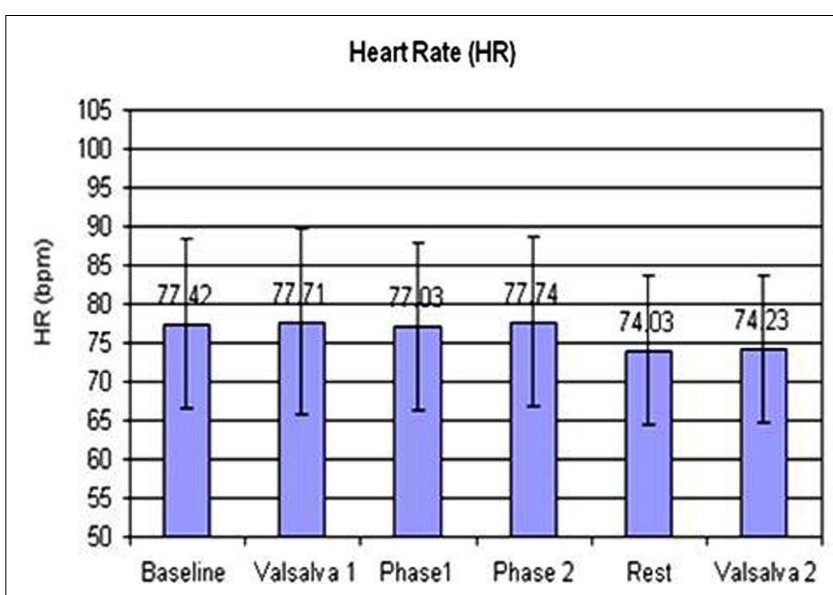

FIGURE 3 | The changes in heart rate (HR) across the tasks.

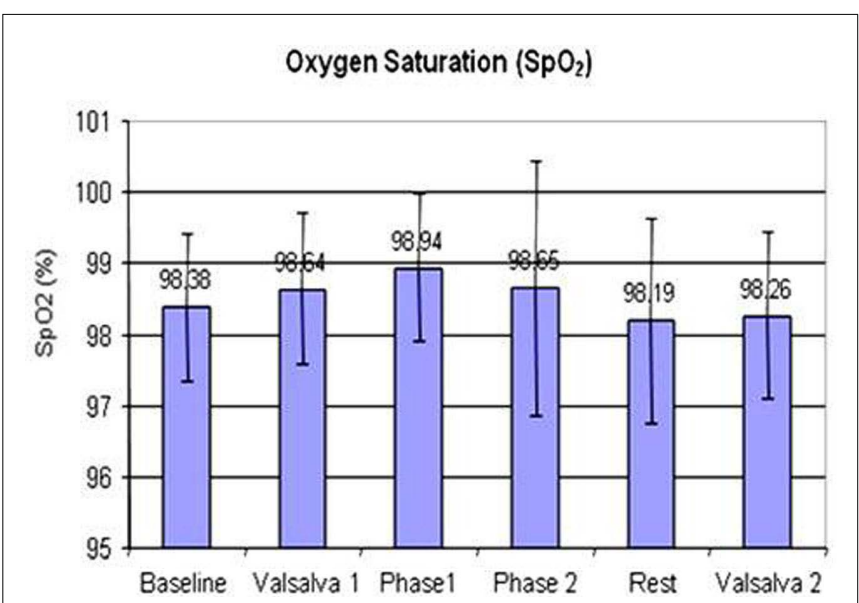

FIGURE 4 |The changes in oxygen saturation $\left(\mathrm{SpO}_{2}\right)$ across the tasks.

participants who generated higher MEP at the baseline and subsequently during the EMST trials showed higher $\mathrm{SpO}_{2}$ at the baseline and through all the tasks of the study session.

Based on the separate analysis for men and women respectively, no significant changes were observed in SBP $(p=0.6357$; $p=0.3188), \operatorname{DBP}(p=0.6755 ; p=0.8351), \operatorname{HR} \quad(p=0.1091$; $p=0.7307)$, and $\mathrm{SpO}_{2}(p=0.6063 ; p=0.2476)$ across the tasks.

\section{DISCUSSION}

This study defined the cardiovascular responses to relatively high expiratory pressures generated with a single session of an EMST device by healthy young adults. The results showed no significant changes in $\mathrm{BP}, \mathrm{HR}$, and $\mathrm{SpO}_{2}$ during and following a session of 25 EMST trials with the EMST device set at 75\% MEP, suggesting little impact on $\mathrm{BP}, \mathrm{HR}$, and $\mathrm{SpO}_{2}$ for the cohort enrolled in the study This outcome supports the hypothesis that EMST would not induce major fluctuations in cardiovascular response primarily because of the brief and repetitive expiratory effort durations 
of the task. Even though a high expiratory pressure is generated to open the valve within the device, the duration of that pressure generation is on the order of $1.25 \mathrm{~s}$. This duration is substantially less than a typical Valsalva maneuver which can last anywhere from 15 to $20 \mathrm{~s}$ or more depending on the task (Luster et al., 1996; Tiecks et al., 1996).

Both Chaudhuri et al. (2002) and the present study analyzed BP and HR changes during brief, repetitive acts of exertion involving a mechanism similar to the maximal Valsalva maneuver. The supersupraglottic and supraglottic swallow exercises described in the Chaudhuri et al. (2002) study evoked significant BP elevation and cardiac arrhythmia. Chaudhuri et al. (2002) reported BP increases ( $>20 \mathrm{mmHg}$ of SBP or $>10 \mathrm{mmHg}$ DBP) in 8 out of 23 older adults (44-90 years of age), including 5 participants with history of coronary artery disease during supraglottic and super-supraglottic swallow maneuvers. Thirteen out of 15 participants with a history of stroke and dysphagia demonstrated cardiac arrhythmia during swallowing sessions using the same technique. In addition, 7 of the 23 participants experienced lightheadedness during the treatment session (Chaudhuri et al., 2002). In the Chaudhuri et al. (2002) study, a Holter monitor was used to record electrical activities of the heart and swallow timing, but the duration of the swallow maneuvers and the baseline measurements of BP and HR were not reported. Compared to the results presented by Chaudhuri et al. (2002), the present study participants demonstrated no significant changes of mean SBP and mean DBP across the tasks.

Reasons for the study results differences are likely the following: first, the swallow supraglottic and super-supraglottic exercises clearly involved "bearing down" against a closed glottis, which was considered a modified Valsalva maneuver (Chaudhuri et al., 2002). While the glottis is narrowing during an EMST trial, it either closes so briefly or never completely closes that a cardiovascular response is insignificant due to the limited exertion. Second, two-thirds of the participants of Chaudhuri et al. (2002) study had a history of stroke and cardiovascular disease which could have elicited the abnormal hemodynamic responses to the Valsalva maneuver. The present study results did not show a significant cardiovascular response to any of the Valsalva maneuvers in the studied group. The Valsalva maneuver was shorter ( $5 \mathrm{~s}$ ) than the typical 15-20s Valsalva maneuver presented in the literature (Luster et al., 1996; Tiecks et al., 1996). No considerable BP, $\mathrm{HR}$, or $\mathrm{SpO}_{2}$ fluctuations were noticed after completing each of the Valsalva maneuvers as

\section{REFERENCES}

Baas, L. S., Meissner, J. E., and Coughlin, A. (2002) "Cardiovascular care," in Illustrated Manual of Nursing Practice, 3rd Edn, ed. H. N. Holmes (Springhouse: Lippincott Williams \& Wilkins), 168-323.

Baker, S., Davenport, P., and Sapienza, C. (2005). Examination of strength training and detraining effects in expiratory muscles. J. Speech Lang. Hear. Res. 48, 1325-1333.

Behazin, N., Jones, S. B., Cohen, R. I., and Loring, S. H. (2010). Respiratory restriction and elevated

compared to the baseline values or the values recorded after EMST phases. Monitoring intrathoracic pressure would have allowed better control over the Valsalva maneuver. Without monitoring individual intrathoracic pressures, the degree of exertion could not be physiologically defined. Measuring the intrathoracic pressure requires an invasive and complicated procedure of inserting an intraesophageal catheter (Behazin et al., 2010) which was beyond the scope of this study.

The effects of $\mathrm{BMI}$ and sex on $\mathrm{BP}, \mathrm{HR}$, and $\mathrm{SpO}_{2}$ observed in this investigation are consistent with well known findings presented in current literature (Johnson et al., 1975; Burt et al., 1995; Janssen et al., 2000; Kazumi et al., 2002). An independent hypothesis for BMI, sex, and MEP effects may be considered in the future investigations stimulated by the results of this study.

\section{CONCLUSION}

The current study found a single session with the EMST device in a healthy cohort of young participants resulted in no significant cardiovascular responses to the breathing task. The results of this study suggest that EMST breathing tasks have less cardiovascular effects than reported previously for a Valsalva maneuver. Furthermore, short durations (5s) Valsalva maneuvers have less cardiovascular effects than previous reports for long duration (greater than $10 \mathrm{~s}$ ) maneuvers. Thus, an EMST task is not equivalent to a standard Valsalva maneuver. It is rather a strong expiratory effort with expiratory airflow at maximal pressures less than a pressure exerted during a Valsalva maneuver. Further study of cardiovascular response during EMST is necessary to generalize the results to patient populations. It is crucial for respiratory rehabilitation exercises to be tested for their safety, particularly for patients who have a complex medical history where exacerbation of symptoms could occur during the rehabilitation process. Thorough information regarding the presence or an absence of side effects during the use of an exercise device may guide professional practice. One caution is that patients with cardiovascular disease can suffer with a multitude of conditions, ranging from hypertension to atherosclerosis which may lead to either cerebral or myocardial infarction. An assessment of specific effects of EMST on these patients is required before considering the use of EMST. Nevertheless, the absence of significant effects of EMST on BP, HR, and $\mathrm{SpO}_{2}$ in the healthy population is promising, and, if this also applies to patients populations, EMST is a safe tool to improve expiratory muscle strength.

effects of the supraglottic and supersupraglottic swallowing maneuvers in stroke patients with dysphagia. Dysphagia 17, 19-23.

Chiara, T., Martin, A. D., Davenport, P. W., and Bolser, D. C. (2006). Expiratory muscle strength training in persons with multiple sclerosis having mild to moderate disability: effect on maximal expiratory pressure, pulmonary function, and maximal voluntary cough. Arch. Phys. Med. Rehabil. 87, 468-473.

Elghozi, J.-L., Girard, A., Fritsch, P., Laude, D., and Petitprez, J.-L. (2008).
Tuba players reproduce a Valsalva maneuver while playing high notes. Clin. Auton. Res. 18, 96-104.

Harraf, F., Ward, K., Man, W., Rafferty, G., Mills, K., Polkey, M., Moxham, J., and Kalra, L. (2008). Transcranial magnetic stimulation study of expiratory muscle weakness in acute ischemic stroke. Neurology 71, 2000-2007.

Janssen, I., Heymsfield, S. B., Wang, Z., and Ross, R. (2000). Skeletal muscle mass and distribution in 468 men and women aged 18-88 yr. J. Appl. Physiol. 89, 81-88. 
Johnson, A. L., Cornoni, J. C., Cassel, J. C., Tyroler, H. A., Heyden, S., and Hames, C. G. (1975). Influence of race, sex and weight on blood pressure behavior in young adults. Am. J. Cardiol. 35, 523-530.

Joint National Committee on Prevention, Detection, Evaluation, and Treatment of High Blood Pressure. (1997). The sixth report of the joint national committee on prevention, detection, evaluation, and treatment of high blood pressure. Arch. Intern. Med. 157, 2413-2446. [Erratum, Arch Intern Med 1998;158:573].

Kazumi, T., Kawaguchi, A., Sakai, K., Hirano, T., and Yoshino, G. (2002). Young men with highnormal blood pressure have lower serum adiponectin, smaller LDL size, and higher elevated heart rate than those with optimal blood pressure. Diabetes Care 25, 971-976.

Kim, J., Davenport, P., and Sapienza, C. (2009). Effect of expiratory muscle strength training on elderly cough function. Arch. Gerontol. Geriatr. 48, 361-366.

Kim, J., and Sapienza, C. M. (2005). Implications of expiratory muscle strength training for rehabilitation of the elderly: tutorial. J. Rehabil. Res. Dev. 42, 211-223.

Levin, A. B. (1966). A simple test of cardiac function based upon the heart rate changes induced by the Valsalva maneuver. Am. J. Cardiol. 18, 90-99.

Luster, E. A., Baumgartner, N., Adams, W. C., and Convertino, V. A. (1996). Effects of hypovolemia and posture on responses to the Valsalva maneuver. Aviat. Space Environ. Med. 67, 308-313.

Manley, G., Knudson, M. M., Morabito, D., Damron, S., Erickson, V., and Pitts, L. (2001). Hypotension, hypoxia, and head injury: frequency, duration, and consequences. Arch. Surg. 136, 1118-1123.

McConnell, A. K., and Romer, L. M. (2004). Respiratory muscle training in healthy humans: resolving the controversy. Int. J. Sports Med. 25, 284-293.

Metzger, B. L., and Therrien, B. (1990). Effect of position on cardiovascular response during the Valsalva maneuver. Nurs. Res. 39, 198-202.

Narloch, J. A., and Brandstater, M. E. (1995). Influence of breathing technique on arterial blood pressure during heavy weight lifting. Arch. Phys. Med. Rehabil. 76, 457-462.

Pitts, T., Bolser, D., Rosenbek, J., Troche, M., Okun, M. S., and Sapienza, C. (2009). Impact of expiratory muscle strength training on voluntary cough and swallow function in Parkinson disease. Chest 135 , 1301-1308.

Sapienza, C. M., Davenport, P. W., and Martin, A. D. (2002). Expiratory muscle training increases pressure support in high school band students. J. Voice 16, 495-501.

Tiecks, F. P., Douville, C., Byrd, S., Lam, A. M., and Newell, D. W. (1996). Evaluation of impaired cerebral autoregulation by the Valsalva maneuver. Stroke 27, 1177-1182.

Troche, M., Okun, M., Rosenbek, J., Musson, N., and Sapienza, C. (2009) Swallow outcomes following intervention with expiratory muscle strength training (Emst) in Parkinson's disease: results of a randomized clinical trial. Dysphagia 24 455-456.

Wingate, J. M., Brown, W. S., Shrivastav, R., Davenport, P., and Sapienza, C.
M. (2007). Treatment outcomes for professional voice users. J. Voice 21, 433-449.

Conflict of Interest Statement: Dr. Sapienza and Dr. Davenport serve as scientific consultants and have a financial relationship with Aspire Products, LLC.

Received: 02 December 2011; accepted: 23 February 2012; published online: 13 March 2012.

Citation: Laciuga H, Davenport $P$ and Sapienza C (2012) The acute effects of a single session of expiratory muscle strength training on blood pressure, heart rate, and oxygen saturation in healthy adults. Front. Physio. 3:48. doi: 10.3389/fphys.2012.00048

This article was submitted to Frontiers in Respiratory Physiology, a specialty of Frontiers in Physiology.

Copyright () 2012 Laciuga, Davenport and Sapienza. This is an open-access article distributed under the terms of the Creative Commons Attribution Non Commercial License, which permits noncommercial use, distribution, and reproduction in other forums, provided the original authors and source are credited. 